\title{
Effectiveness of Postoperative Pain Management By Multimodal Analgesia Without Epidural Analgesia After Laparoscopic Colon Cancer Surgery: A Prospective Cohort Study
}

Yoshinori Yane ( $\nabla$ yaneyoshinori0127@med.kindai.ac.jp )

Kindai University

Koji Daito

Kindai University

Yasutaka Chiba

Kindai University

Toru Shirai

Kindai University

Jin-ichi Hida

Kindai University Nara Hospital

Kazuki Ueda

Kindai University

Shinichi Nakao

Kindai University

Junichiro Kawamura

Kindai University

\section{Research Article}

Keywords: Multimodal analgesia, Postoperative pain management, Colorectal cancer, Laparoscopic surgery, Colorectal surgery

Posted Date: December 29th, 2021

DOI: https://doi.org/10.21203/rs.3.rs-1054738/v1

License: (9) (1) This work is licensed under a Creative Commons Attribution 4.0 International License. Read Full License 


\section{Abstract}

\section{Background:}

Although epidural analgesia has been recommended for its strong analgesic effect for postoperative analgesia management, the increasing number of patients undergoing anticoagulant or antiplatelet therapy to treat cerebrocardiovascular diseases cannot receive epidural analgesia given the risk of serious complications, including epidural hematoma. We aimed to evaluate the analgesic effects of multimodal analgesia involving intravenous patient-controlled analgesia (IV-PCA), and repeated scheduled acetaminophen administration, and block as local anesthesia, to establish postoperative analgesia management method replacing epidural analgesia in laparoscopic colectomy.

\section{Methods:}

We enrolled patients undergoing laparoscopic surgery for colorectal cancer at our hospital. The primary outcome was days of postoperative hospital stay. The efficacies of multimodal and epidural analgesia were compared. The secondary outcomes were the pain assessment and safety.

\section{Results:}

We registered 48 patients; among them, 40 patients were eligible. The mean postoperative hospital stay was 9.00 days $(95 \% \mathrm{Cl}=8.19$ to $9.39, \mathrm{p}<0.0001)$. There were relatively high pain scores from postoperative day (POD) 0-1, which subsequently decreased and reach their lowest value at POD 4-5.

\section{Conclusions:}

Multimodal analgesia with IV-PCA and repeated scheduled acetaminophen administration could provide a safe and effective analgesic effect after laparoscopic colectomy and may be a postoperative analgesia management alternative to epidural analgesia.

\section{Background}

Open surgery is conventionally applied for colon cancer surgery with Jacobs reporting the first case of laparoscopic colorectal resection in 1991 [1]. In Japan, there has been an increase in the use of the laparoscopic approach since its first report in 1992 [2]. The advantages of laparoscopic surgery include early post-surgery recovery with reports indicating it involves fewer postoperative hospitalization days compared to open surgery [1]. Further, there have been efforts to achieve early recovery at several facilities through enhanced recovery after surgery (ERAS), which is an evidence-based multidisciplinary program that integrates elements necessary for early recovery. ERAS protocol has significant advantages in open colectomy; moreover, continuous epidural analgesia is recommended for its strong analgesic effect and early bowel functional recovery [3]. Postoperative pain management is an important factor for early postoperative ambulation and recovery; however, epidural analgesia requires catheter placement and could negatively affect early postoperative ambulation. In addition, epidural analgesia requires an 
invasive procedure where a thick needle is punctured into the epidural space under local anesthesia, which could cause fear among patients. Furthermore, patients undergoing anticoagulant or antiplatelet therapy to treat cerebrocardiovascular diseases, which has recently been increasing, cannot receive epidural analgesia given the risk of serious complications, including epidural hematoma.

Intravenous patient-controlled analgesia (IV-PCA) with opioids is a method of pain management that allows postoperative patients to easily secure the administration route. Moreover, it can be used even in patients unable to receive epidural analgesia. However, it has an inadequate analgesic effect during coughing and body movement $[4,5]$. Furthermore, additional bolus opioid administration could be required in uncontrolled patients, which might induce opioid-related side effects [6]. Therefore, there is a need for a rational approach involving various treatment modalities to obtain optimal pain control and minimize opioid use. Multimodal analgesia, which combines different analgesic drug classes, is recommended for postoperative pain treatment since its synergistic effect on pain relief at lower analgesic doses [7].

Unlike open surgery, laparoscopic surgery has a high risk of mild pain and often requires alternative pain management to epidural analgesia. Given the increasing adoption of laparoscopic surgery for colorectal cancers, there is a need to establish postoperative pain management for replacing epidural analgesia. We aimed to evaluate the feasibility of IV-PCA and repeated scheduled acetaminophen administration to establish an alternative postoperative analgesia management protocol to epidural analgesia in laparoscopic colectomy.

\section{Methods}

\section{Patients and eligible criteria}

The inclusion criteria were as follows: patients aged 20-85 years old; having histologically confirmed colorectal cancer located at the colon or rectosigmoid junction; undergone laparoscopic colectomy at our hospital; having adequate critical organ function; and having an Eastern Cooperative Oncology Group performance status 0 or 1 . We obtained written informed consent from all patients at study enrollment. The exclusion criteria were as follows: having rectal cancer and distant metastases; being allergic to any drugs used in this study; and having an active infectious disease, as well as uncontrolled diabetes mellitus, hypertension, and urinary disorder. The study was conducted in accordance with the Helsinki declaration on experimentation on human subjects. Further, this study was approved by the Institutional Ethical Review Boards of Kindai University (approval no. 29-033) and registered at the UMIN Clinical Trials Registry as UMIN000028240, 14/07/2017 (http://www.umin.ac.jp/ctr/index-j.htm).

\section{Intraoperative pain management}

General anesthesia induction was performed using propofol, remifentanil, and rocuronium. Further, intraoperative anesthesia management was performed using desflurane or sevoflurane, remifentanil, and additional rocuronium. Additionally, at the time of closure before surgery end, $20 \mathrm{~mL}$ of $0.375 \%$ 
ropivacaine (Anapeine ${ }^{\circledR 囚}$ Injection; AstraZeneca, Osaka, Japan) was administered onto the fascia of each wound as local anesthesia.

\section{Postoperative pain management}

At the end of surgery, a solution of fentanyl $(25 \mu \mathrm{g} / \mathrm{mL})$ and droperidol $(0.042 \mathrm{mg} / \mathrm{mL})$ was administered at a rate of $1 \mathrm{ml} / \mathrm{h}$ using portable infusing devices (Syrinjector ${ }^{\circledR \mathbb{R}}$ ) until the morning of POD2. A $1 \mathrm{~mL}$ bolus was allowed at a lockout interval of 30 minutes if the patients wanted more analgesia.

Furthermore, $1000 \mathrm{mg}$ of acetaminophen intravenous solution (Acelio ${ }^{\circledR}$ Intravenous Injection; Terumo Corporation, Tokyo, Japan) was administered at the end of the surgery and subsequently at 6-h intervals until POD 3. In addition, the use of rescue analgesic drugs, including $50 \mathrm{mg}$ flurbiprofen axetil (i.v.; Ropion ${ }^{\circledR 囚}$; Kaken Phamaceutical, Tokyo, Japan) were permitted as needed.

\section{Outcome measures}

The primary endpoint was days of postoperative hospital stay. The secondary endpoints included the numerical rating scale (NRS) scores, prince Henry pain scale (PRS) scores, and safety. A nurse performed pain evaluation using the NRS and PRS at 8-hour intervals from 6 hours after surgery until POD5, as well as at first ambulation. Further, the frequency of the use of PCA (bolus administration) and rescue analgesic drugs was documented.

The NRS is an 11-point scale with " 0 " representing "no pain" and " 10 " representing "most severe pain imaginable". The PRS is a $0-4$ scale with $0,1,2,3$, and 4 indicating no pain at coughing, pain at coughing but not at deep breathing, pain at deep breathing but not at rest, pain at rest not requiring analgesics, and pain at rest requiring analgesics, respectively. Adverse events were evaluated using Common Terminology Criteria for Adverse Events (CTCAE) Ver.4.0. Postoperative complications were evaluated based on the evaluation classification of Clavian-Dindo.

\section{Statistical analysis}

The primary endpoint was days of postoperative hospital stay. There were 531 patients who underwent epidural analgesia for laparoscopic colectomy at our hospital between 2005 and 2015. The distribution of hospital days was skewed to the right, which indicates a long hospital stay. Subsequently, we analyzed the data on a log scale. The mean postoperative hospital stay was 2.310 (standard deviation [SD] = 0.378) on the log scale, where $\exp (2.310)=10.073$ days. Based on the aforementioned findings, we assumed that the expected postoperative hospital stay using multimodal analgesia was $2.303(\mathrm{SD}=0.4)$ on the log scale, where exp $(2.303)=10$ days. Based on these assumptions, this study was designed to examine the non-inferiority of multimodal analgesia compared with epidural analgesia using a 2-day margin. Based on a significance level of 0.025 (one-sided) and power of 0.8, a one-sample t-test showed that 40 patients were required. To account for patients ineligible for analysis, we enrolled 45 patients with the analysis set being patients with available records during the days of postoperative hospital stay. The results were presented after converting the mean and calculating the $95 \%$ confidence interval $(\mathrm{Cl})$ on the log scale to the exponential scale, with the one-sided p-value. 
The secondary endpoints were the NRS score, PRS score, and safety. The NRS and PRS scores were summarized using box-whisker plots; moreover, the frequency of adverse events was determined.

All statistical analysis was performed using JMP pro15 (SAS Institute Inc., Cary, NC, USA).

\section{Results}

\section{Demographics}

Between September 2017 and August 2018, we performed 73 laparoscopic colectomies with 48 patients being registered and 40 of them being eligible. The 8 excluded patients included 3 with rectum lesions, 2 with resection of other organs, 2 with inappropriate intraoperative drug usage, and 1 who could not undergo postoperative evaluation due to dementia (Figure 1).

\section{Clinical characteristics}

Table 1 presents the clinical characteristics of the patients. There were 22 males and 18 females. The median age was 73.5 years (range: 55-85 years). The median body mass index was $23.2 \mathrm{~kg} / \mathrm{m}^{2}$ (range: $\left.15.0-30.1 \mathrm{~kg} / \mathrm{m}^{2}\right)$. Eight $(20 \%)$ patients had a history of diabetes mellitus while six $(15 \%)$ patients had perioperative heparin substitution. The tumor location was the sigmoid colon in $16(40 \%)$ patients, the ascending colon in $8(20 \%)$ patients, the cecum in $7(17.5 \%)$ patients, the rectosigmoid in $5(12.5 \%)$ patients, the transverse colon in $3(7.5 \%)$ patients, and the descending colon in $1(2.5 \%)$ patient. Table 2 presents the surgical procedures and short-term outcomes. Among the patients with colorectal cancers, 6 , $10,3,13$, and 8 patients underwent ileocecal resection, right hemicolectomy, left hemicolectomy, sigmoidectomy, and high anterior resection, respectively. D3 and D2 lymph node dissections were performed in $35(87.5 \%)$ and $5(12.5 \%)$ patients, respectively. Functional end-to-end anastomosis was performed in 18 patients $(45.0 \%)$ while the double stapling technique was performed in $22(55.0 \%)$ patients. The median surgery duration, bleeding volume, induction time, and time spent in the operation room were 202 minutes (range: 139-317 minutes), $10 \mathrm{ml}$ (range: 10-300 ml), 48 minutes (range: 35-89 minutes), and 289.5 minutes (range: 211-424 minutes), respectively. 
Table 1

Clinical characteristics of the patients

\begin{tabular}{|ll|}
\hline & Total(n=40) \\
\hline Age(years), median(range) & $73.5(55 \sim 85)$ \\
\hline Sex, $\mathrm{n}(\%)$ & \\
\hline Male & $22(55)$ \\
\hline Female & $18(45)$ \\
\hline BMI(kg/m²), median(range) & $23.2(15.0 \sim 30.1)$ \\
\hline Diabetes mellitus, $\mathrm{n}(\%)$ & $8(20)$ \\
\hline Heparin substitution, $\mathrm{n}(\%)$ & $6(15)$ \\
\hline Location of the tumor, $\mathrm{n}(\%)$ & \\
\hline Cecum & $7(17.5)$ \\
\hline Ascending & $8(20)$ \\
\hline Transverse & $3(7.5)$ \\
\hline Descending & $1(2.5)$ \\
\hline Sigmoid & $16(40)$ \\
\hline Rectosigmoid & $5(12.5)$ \\
\hline BMl, body mass index & \\
\hline
\end{tabular}


Table 2

Surgical procedures and outcomes

\begin{tabular}{|ll|}
\hline & Total(n=40) \\
\hline Surgical procedures, $\mathrm{n}(\%)$ & \\
\hline Ileocecal resection & $6(15)$ \\
\hline Right hemicolectomy & $10(25)$ \\
\hline Left hemicolectomy & $3(7.5)$ \\
\hline Sigmoidectomy & $13(32.5)$ \\
\hline High anterior resection & $8(20)$ \\
\hline Lymph node dissection, $\mathrm{n}(\%)$ & \\
\hline D2 & $5(12.5)$ \\
\hline D3 & $35(87.5)$ \\
\hline Anastomosis method, $n(\%)$ & $18(45)$ \\
\hline Functional end-to-end anastomosis & $22(55)$ \\
\hline Double stapling technique anastomosis & \\
\hline Surgical outcomes & $202(139-317)$ \\
\hline Surgery duration, minutes, median(range) & $10(10-300)$ \\
\hline Bleeding volume, ml, median(range) & $48(35-89)$ \\
\hline Anesthesia induction time, minutes, median(range) & $289.5(211-424)$ \\
\hline Operation room time, minutes, median(range) & \\
\hline Postoperative complications, $n(\%)$ & $1(2.5)$ \\
\hline Nausea & \\
\hline Vomiting & \\
\hline Liver dysfunction & \\
\hline Lower gastrointestinal bleeding & \\
\hline
\end{tabular}

\section{Postoperative hospital stay and pain evaluation}

The mean postoperative hospital stay was 9.00 days $(95 \% \mathrm{Cl}=8.19$ to $9.39, \mathrm{p}<0.0001)$, which indicates that the primary endpoint was met.

Postoperative pain was evaluated between POD0 and POD5 using the NRS and PRS (Fig. 2). There were relatively high NRS and PRS scores from POD0-1, which subsequently decreased and reached their 
lowest values from POD4-5. During the observation period, 10 (25\%) patients used PCA while 2 (5\%) patients used adjuvant analgesics.

\section{Postoperative ambulation and oral intake}

The median time to ambulation and first oral intake was 1 day (range: 1-3 days) and 4 days (range: 4-5 days), respectively.

\section{Short-term complications}

Table 1 shows the postoperative complications. Nausea and vomiting occurred in 17 (42.5\%) and 12 (30.0\%) patients, respectively. Three (7.5\%) patients discontinued IV-PCA due to nausea and vomiting. Liver dysfunction occurred in 7 (17.5\%) patients with increased aspartate aminotransferase (AST) or alanine aminotransferase (ALT) levels (CTCAE v4.0 $\geq$ Grade 1) with all of them recovering rapidly. One $(2.5 \%)$ patient presented with lower gastrointestinal bleeding (Clavien-Dindo classification $\geq$ Grade 2 ). There were no serious postoperative complications, including dysuria, respiratory depression, hypotension, allergic reactions, and asthma attacks.

\section{Discussion}

Our findings indicated that IV-PCA and repeated scheduled acetaminophen administration has an efficient analgesic effect as postoperative analgesia after laparoscopic colectomy. The IV-PCA and acetaminophen combination maintained a low pain score and did not affect the postoperative hospital stay. In addition, repeated scheduled acetaminophen administration exerted a persistent analgesic effect and reduced the frequency of the fentanyl bolus dose and unnecessary additional opioid administration. Furthermore, only two patients required additional use of non-opioid analgesics.

Epidural analgesia is considered as a very useful postoperative analgesic method for postoperative recovery [6, 8-10]. However, as an epidural block, it involves potential technical and analgesic risks, including epidural hematoma, hypotension, postoperative urination disorder, limb paresthesia, and limb movement disorder. Moreover, it involves some risks associated with epidural opioids, including nausea, vomiting, and pruritus [8, 11-15]. Specifically, epidural hematoma has become a common severe adverse effect given the recent increase in the number of patients receiving anticoagulant therapy [16].

The single administration or patient-controlled analgesia (IV-PCA) with fentanyl is widely used as a postoperative analgesia alternative to epidural analgesia. However, compared to epidural analgesia, IVPCA is considered to have an insufficient analgesic effect, especially during coughing and body movement $[4,5]$. Patients using IV-PCA with postoperative uncontrollable pain could require additional bolus administration, as well as develop opioid side effects; specifically, postoperative nausea and vomiting (PONV), which is dose dependent [17]. Compared with placebo, repeated scheduled acetaminophen administration, i.e., 2 doses of intravenous acetaminophen (1000 mg q6h and $650 \mathrm{mg}$ $\mathrm{q} 4 \mathrm{~h}$ ), has been shown to have a significant analgesic effect after abdominal laparoscopic surgery [18]. 
Consequently, we speculated that IV-PCA and repeated scheduled acetaminophen administration could provide a sufficient analgesic effect and opioid side effects could be relieved by reducing the opioid dose.

Regarding the adverse effects of analgesics, PONV is among the major adverse effects and could cause extreme distress to postoperative patients. PONV is associated with prolonged length of postoperative hospital stay given the delayed first oral intake, which concomitantly leads to high medical costs [19]. PONV is usually caused by intraoperative volatile anesthetics and postoperative opioids [20]. Postoperative opioids increase the risk of PONV in a dose-dependent manner with the effect remaining throughout the opioid usage period for pain control in the postoperative periods [17, 21]. The volatile anesthetic effect on PONV is specifically prominent during the first 2-6 postoperative hours [20]. There are some risk factors for PONV, including female gender, non-smoking status, a history of PONV, a history of motion sickness, young age, laparoscopic surgery, and gynecological surgery [22]. Several drugs can be used for PONV, including 5-hydroxytryptamine and neurokinin-1 receptor antagonists, corticosteroids, butyrophenones, antihistamines, anticholinergics, etc. [22]. In our study, 17 (42.5\%) patients (12 were women) presented with fentanyl-caused PONV after postoperative day 1 even with droperidol interfusion in IV-PCA. Future studies should discuss PONV prevention and treatment after laparoscopic colectomy.

Compared with epidural anesthesia, IV-PCA could induce other adverse effects, including respiratory depression [23] and delayed bowel movement [4, 8, 24]. Although properly applied IV-PCA is safe in most postoperative patients, some patients experience severe respiratory depression due to frequent additional bolus fentanyl, which requires opioid antagonists. We did not observe any cases of respiratory depression. Repeated scheduled acetaminophen administration could reduce the fentanyl dose and significantly contributes to reduced adverse events.

We preferred using acetaminophen, rather than NSAIDs, as a postoperative analgesic since NSAIDs increase the risk of gastrointestinal ulcers, renal toxicity, and anastomotic leakage $[25,26]$. There is no association of short-term therapeutic use of acetaminophen with adverse effects, including bleeding, renal toxicity, and gastrointestinal disorders $[27,28]$. This indicates that repeated acetaminophen administration is safer than repeated NSAID administration. Acetaminophen is a relatively safe drug; however, it could cause several adverse effects with liver dysfunction being the most adverse side effects [29]. In our study, 7 patients presented with liver dysfunction characterized by elevated AST or ALT levels (CTCAE Grade 1), which subsequently rapidly improved. Therefore, repeated acetaminophen administration is feasible in most patients without chronic liver damage.

Epidural analgesia has some potential disadvantages other than the aforementioned technical risks. The analgesic effect of epidural analgesia could be significantly dependent on the anesthesiologist's skill. Moreover, even with proper insertion, an analgesic effect may not be attained. Furthermore, the epidural technique can be technically difficult and time consuming even when properly performed [15]. Contrastingly, IV-PCA lacks technical difficulties cannot be safely performed by non-experts. Moreover, it reduces the operating room time. 
This study has several limitations. First, it analyzed a small sample size. In addition, we did not directly compare the outcomes between epidural and multimodal analgesia. Instead, we compared the outcomes between the study group and a previously analyzed historical group as the epidural analgesia group. There were some clinical between-group differences, including the period backgrounds.

\section{Conclusions}

Our findings suggest that continuous intravenous fentanyl administration and repeated scheduled acetaminophen administration could provide a safe and efficient analgesic effect in laparoscopic colectomy. There is a need for future prospective comparative studies to compare between epidural and multimodal analgesia.

\section{Declarations}

\section{Ethics approval:}

All authors comply that this research was conducted ethically in accordance with the World Medical Association Declaration of Helsinki. Further, this study was approved by the Institutional Ethical Review Boards of Kindai University (approval no. 29-033) and registered at the UMIN Clinical Trials Registry as UMIN000028240, 14/07/2017 (http://www.umin.ac.jp/ctr/index-j.htm). Written informed consent were obtained from all patients at study enrollment.

\section{Consent to participate:}

Written informed consent was obtained from all individual participants included in the study.

\section{Consent for publication:}

All authors have approved the final version of the manuscript and agree with submission to $B M C$ Anesthesiology.

\section{Data Availability Statement:}

The data that support the findings of this study are available from the corresponding author, YY, upon reasonable request.

\section{Conflicts of interests/ Competing interests:}

The authors have no conflicts interest to disclose.

\section{Funding:}

This research did not receive any specific grant from funding agencies in the public, commercial, or notfor-profit sectors. 


\section{Author Contributions:}

All authors contributed to the study conception and design. Material preparation and data collection were performed by $Y Y$ and KD. Analysis and interpretation of data were performed by YY, YC, and JK. The first draft of the manuscript was written by YY. KD, TS, JH, KU, SNand JK commented on previous versions of the manuscript. All authors read and approved the final manuscript.

\section{Acknowledgments:}

We would like to thank Editage (www.editage.com) for English language editing.

Code availability: Not applicable

\section{References}

1. Schwenk W, Haase O, Neudecker J, Müller JM. Short term benefits for laparoscopic colorectal resection. Cochrane Database Syst Rev. 2005;3:CD003145. https://doi.org/10.1002/14651858.CD003145.pub2

2. Kitano S, Kitajima M, Konishi F, Kondo H, Satomi S, Shimizu N. A multicenter study on laparoscopic surgery for colorectal cancer in Japan. Surg Endosc. 2006;20:1348-1352. https://doi.org/10.1007/s00464-004-8247-9

3. Fearon KC, Ljungqvist $\mathrm{O}$, Von Meyenfeldt $\mathrm{M}$, et al. Enhanced recovery after surgery: a consensus review of clinical care for patients undergoing colonic resection. Clin Nutr. 2005;24:466-477. https://doi.org/10.1016/j.clnu.2005.02.002

4. Carli F, Trudel JL, Belliveau P. The effect of intraoperative thoracic epidural anesthesia and postoperative analgesia on bowel function after colorectal surgery: a prospective, randomized trial. Dis Colon Rectum. 2001;44:1083-1089. https://doi.org/10.1007/bf02234626

5. Pirec V, Laurito CE, Lu Y, Yeomans DC. The combined effects of N-type calcium channel blockers and morphine on A delta versus C fiber mediated nociception. Anesth Analg. 2001;92:239-243. https://doi.org/10.1097/00000539-200101000-00046

6. Nishimori M, Ballantyne JC, Low JH. Epidural pain relief versus systemic opioid-based pain relief for abdominal aortic surgery. Cochrane Database Syst Rev. 2006;3:CD005059. https://doi.org/10.1002/14651858.CD005059.pub2

7. Garimella V, Cellini C. Postoperative pain control. Clin Colon Rectal Surg. 2013;26:191-196. http://doi.org/10.1055/s-0033-1351138

8. Werawatganon T, Charuluxanun S. Patient controlled intravenous opioid analgesia versus continuous epidural analgesia for pain after intra-abdominal surgery. Cochrane Database Syst Rev. 2005;1:CD004088, https://doi.org/10.1002/14651858.CD004088.pub2

9. Kehlet H, Holte K. Effect of postoperative analgesia on surgical outcome. Br J Anaesth. 2001;87:6272. 
10. Ganapathi S, Roberts G, Mogford S, Bahlmann B, Ateleanu B, Kumar N. Epidural analgesia provides effective pain relief in patients undergoing open liver surgery. Br J Pain. 2015;9:78-85. https://doi.org/10.1177/2049463714525140

11. Wulf H. Epidural anaesthesia and spinal haematoma. Can J Anaesth. 1996;43:1260-1271.

12. Mann C, Pouzeratte Y, Boccara G, et al. Comparison of intravenous or epidural patient-controlled analgesia in the elderly after major abdominal surgery. Anesthesiology. 2000;92:433-441.

13. Bromage PR, Camporesi EM, Durant PA, Nielsen $\mathrm{CH}$. Nonrespiratory side effects of epidural morphine. Anesth Analg. 1982;61:490-495.

14. Liu SS, Allen HW, Olsson GL. Patient-controlled epidural analgesia with bupivacaine and fentanyl on hospital wards: prospective experience with 1,030 surgical patients. Anesthesiology. 1998;88:688695. https://doi.org/10.1097/00000542-199803000-00020

15. Pöpping DM, Zahn PK, Van Aken HK, Dasch B, Boche R, Pogatzki-Zahn EM. Effectiveness and safety of postoperative pain management: a survey of 18925 consecutive patients between 1998 and 2006 (2nd revision): a database analysis of prospectively raised data. Br J Anaesth. 2008;101:832-840. https://doi.org/10.1093/bja/aen300

16. Geerts WH, Bergqvist D, Pineo GF, et al. Prevention of venous thromboembolism: American College of Chest Physicians Evidence-Based Clinical Practice Guidelines (8th Edition). Chest. 2008;133:381-453. https://doi.org/10.1378/chest.08-0656

17. Roberts GW, Bekker TB, Carlsen HH, Moffatt CH, Slattery PJ, McClure AF. Postoperative nausea and vomiting are strongly influenced by postoperative opioid use in a dose-related manner. Anesth Analg. 2005;101:1343-1348. https://doi.org/10.1213/01.ANE.0000180204.64588.EC

18. Wininger SJ, Miller H, Minkowitz HS, Royal MA, Ang RY, Breitmeyer JB, Singla NK. A randomized, double-blind, placebo-controlled, multicenter, repeat-dose study of two intravenous acetaminophen dosing regimens for the treatment of pain after abdominal laparoscopic surgery. Clin Ther. 2010;32:2348-2369. https://doi.org/10.1016/j.clinthera.2010.12.011

19. Ferguson SE, Malhotra T, Seshan VE, et al. A prospective randomized trial comparing patientcontrolled epidural analgesia to patient-controlled intravenous analgesia on postoperative pain control and recovery after major open gynecologic cancer surgery. Gynecol Oncol. 2009;114:111116. https://doi.org/10.1016/j.ygyno.2009.03.014

20. Apfel CC, Kranke P, Katz MH, et al. Volatile anaesthetics may be the main cause of early but not delayed postoperative vomiting: a randomized controlled trial of factorial design. Br J Anaesth. 2002;88:659-668. https://doi.org/10.1093/bja/88.5.659

21. Apfel CC, Philip BK, Cakmakkaya OS, et al. Who is at risk for postdischarge nausea and vomiting after ambulatory surgery? Anesthesiology. 2012;117:475-486. https://doi.org/10.1097/ALN.0b013e318267ef31

22. Gan TJ, Diemunsch P, Habib AS, et al. Consensus guidelines for the management of postoperative nausea and vomiting. Anesth Analg. 2014;118:85-113. https://doi.org/10.1213/ANE.0000000000000002 
23. Cashman JN, Dolin SJ. Respiratory and haemodynamic effects of acute postoperative pain management: evidence from published data. Br J Anaesth. 2004;93:212-223. https://doi.org/10.1093/bja/aeh180

24. Hong X, Mistraletti G, Zandi S, Stein B, Charlebois P, Carli F. Laparoscopy for colectomy accelerates restoration of bowel function when using patient controlled analgesia. Can J Anaesth. 2006;53:544550. https://doi.org/10.1007/BF03021843

25. Davies NM, McLachlan AJ, Day RO, Williams KM. Clinical pharmacokinetics and pharmacodynamics of celecoxib: a selective cyclo-oxygenase-2 inhibitor. Clin Pharmacokinet. 2000;38.225-242. https://doi.org/10.2165/00003088-200038030-00003

26. Bhangu A, Singh P, Fitzgerald JE, Slesser A, Tekkis P. Postoperative nonsteroidal anti-inflammatory drugs and risk of anastomotic leak: meta-analysis of clinical and experimental studies. World J Surg. 2014;38:2247-2257. https://doi.org/10.1007/s00268-014-2531-1

27. Lanas A. Nonsteroidal antiinflammatory drugs and cyclooxygenase inhibition in the gastrointestinal tract: a trip from peptic ulcer to colon cancer. Am J Med Sci. 2009;338:96-106. https://doi.org/10.1097/MAJ.0b013e3181ad8cd3

28. John R, Herzenberg AM. Renal toxicity of therapeutic drugs. J Clin Pathol. 2009;62:505-515. https://doi.org/10.1136/jcp.2008.058271

29. Watkins PB, Kaplowitz N, Slattery JT, et al. Aminotransferase elevations in healthy adults receiving 4 grams of acetaminophen daily: a randomized controlled trial. JAMA. 2006;296:87-93. https://doi.org/10.1001/jama.296.1.87

\section{Figures}




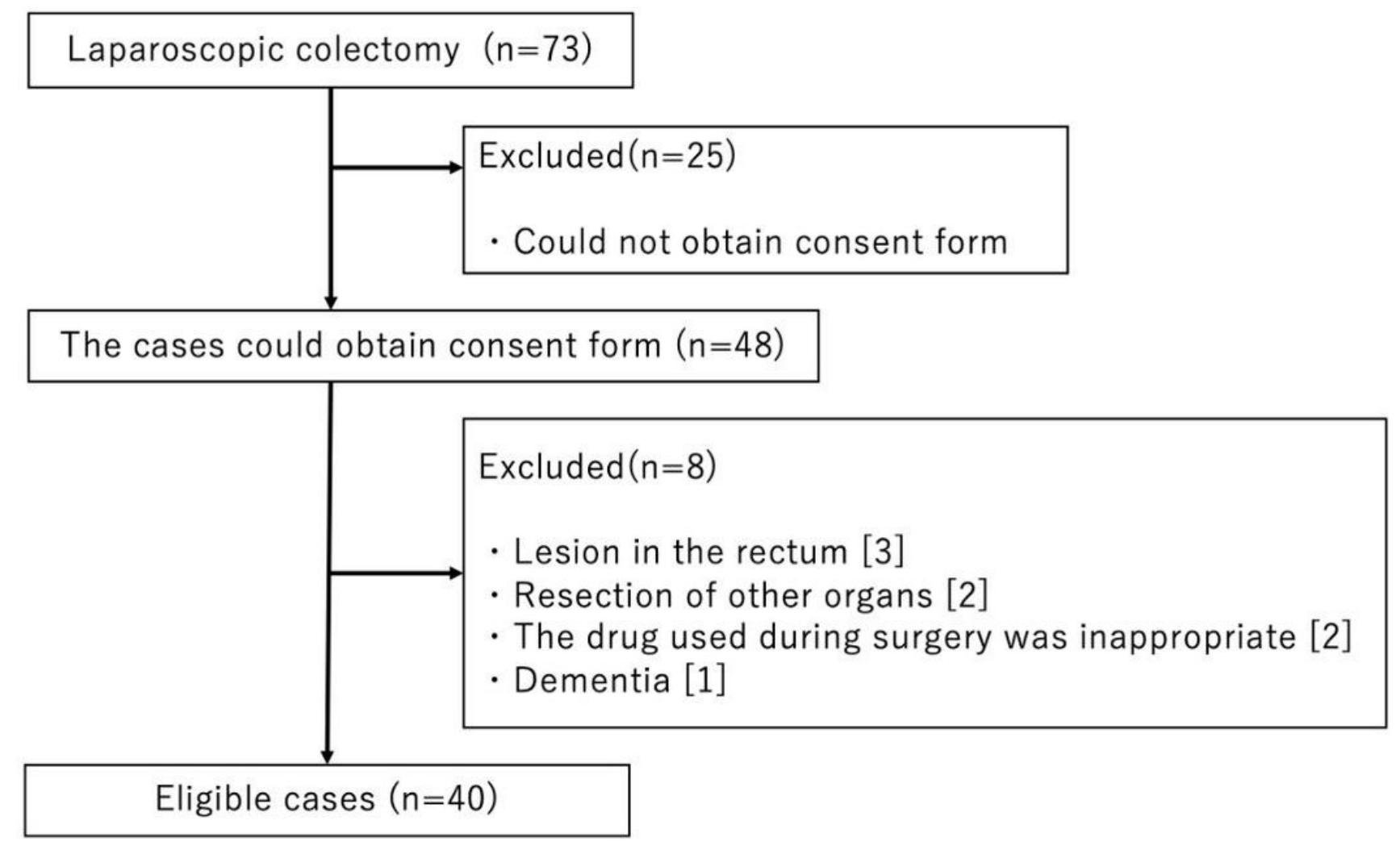

Figure 1

Selection of Study Patients. 

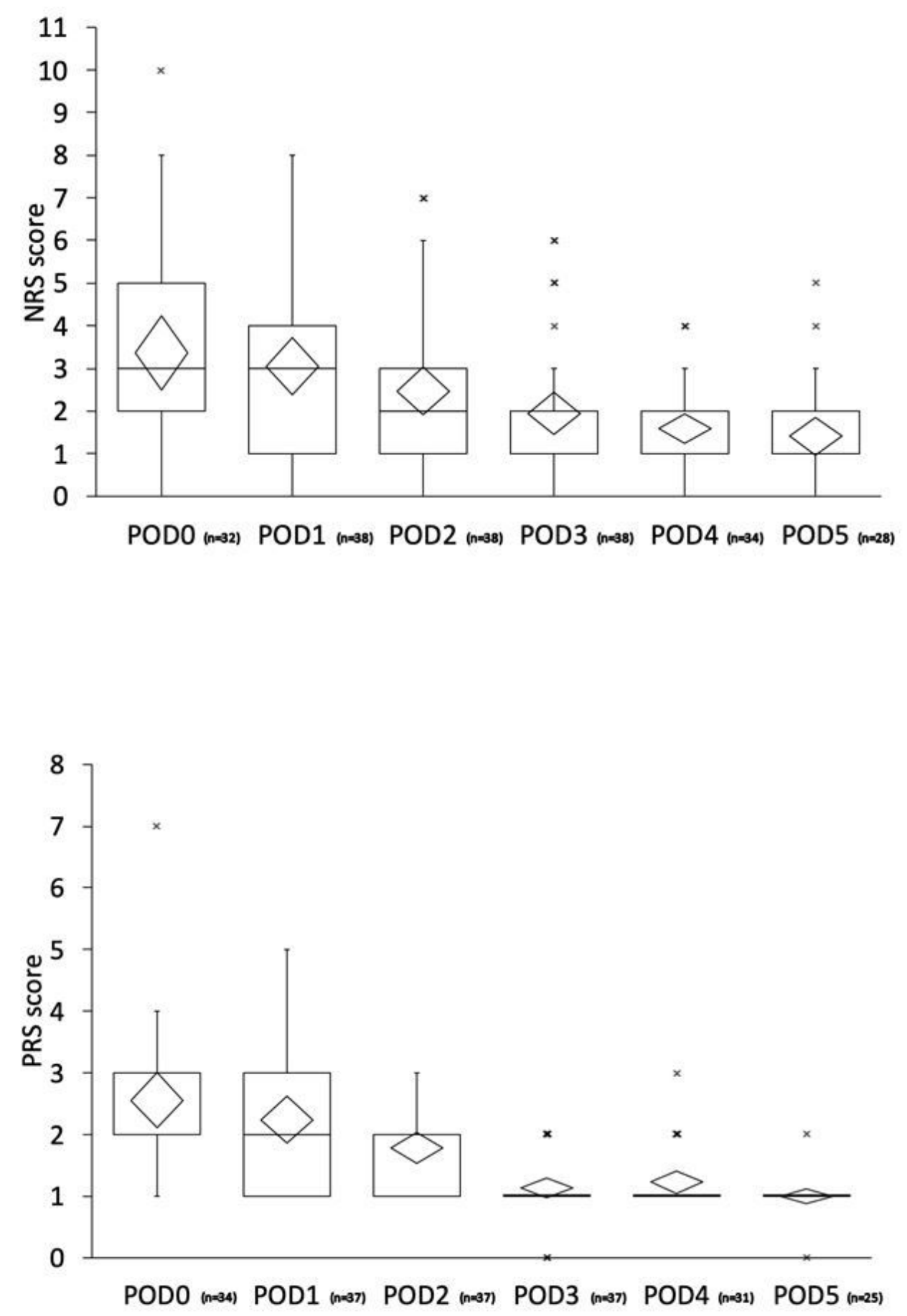

Figure 2

Postoperative pain assessment with NRS score (A) and PRS score (B). NRS score were used 11-points scale and PRS score were used 5-points scale. These scales were observed every 8 hours from 6 hour after surgery to POD5. The box plots show the median, the inter-quartile range, and the confidence interval. 


\section{Supplementary Files}

This is a list of supplementary files associated with this preprint. Click to download.

- Protocol.docx 